\title{
Stopping Antiepileptic Drugs: When and Why?
}

\section{John D. Hixson, MD}

\author{
Address \\ University of California San Francisco, 400 Parnassus Avenue, San Francisco, \\ CA 94143, USA \\ Email: John.hixson@ucsf.edu \\ Published online: 26 June 2010 \\ (C) The Author(s) 2010. This article is published with open access at Springerlink.com
}

\section{Opinion statement}

After a patient has initiated an antiepileptic drug (AED) and achieved a sustained period of seizure freedom, the bias towards continuing therapy indefinitely can be substantial. Studies show that the rate of seizure recurrence after AED withdrawal is about two to three times the rate in patients who continue AEDs, but there are many benefits to AED withdrawal that should be evaluated on an individualized basis. AED discontinuation may be considered in patients whose seizures have been completely controlled for a prolonged period, typically 1 to 2 years for children and 2 to 5 years for adults. For children, symptomatic epilepsy, adolescent onset, and a longer time to achieve seizure control are associated with a worse prognosis. In adults, factors such as a longer duration of epilepsy, an abnormal neurologic examination, an abnormal EEG, and certain epilepsy syndromes are known to increase the risk of recurrence. Even in patients with a favorable prognosis, however, the risk of relapse can be as high as $20 \%$ to $25 \%$. Before withdrawing AEDs, patients should be counseled about their individual risk for relapse and the potential implications of a recurrent seizure, particularly for safety and driving.

\section{Introduction}

After a patient experiences a seizure, there is a natural inclination to intervene. Although a number of variables influence the decision to start an antiepileptic drug (AED), a strong desire to prevent further seizures often tips the balance in favor of a medication. The next question faced by both the patient and the physician is the length of time that treatment is needed. Unlike other medical therapies, which may have well-defined durations, the required duration of antiepileptic therapy is much less concrete and remains somewhat controversial.

This review explores the literature related to the withdrawal of AEDs, focusing on studies and reviews of epilepsy patients who have experienced a prolonged period of seizure freedom. With rare exception, patients who continue to experience seizures require continued therapy. For patients who have entered a period of seizure remission, however, decision-making is more uncertain; AED therapy may have prevented a recurrence of seizures in some patients, but others may have entered a period of seizure remission regardless of therapy. Thus, it is the physician's responsibility to weigh the risks and benefits of continued AED therapy on an individualized basis. This review discusses the factors to be considered in making this decision.

\section{Deciding to Initiate an AED}

Although this review focuses on the decision to withdraw an AED after a seizure-free interval, it is important to acknowledge a number of studies and reviews that 
have addressed the question of starting therapy $[1,2,3,4,5,6,7,8,9]$. After two unprovoked seizures, the risk of recurrence is relatively high [2], a diagnosis of epilepsy is made, and the accepted practice is to initiate an AED. Instituting medical therapy after a single unprovoked seizure event is more controversial. Many studies have examined this issue $[1,2,4,6,7,8]$. Across studies designed as randomized, controlled trials, observational analyses, and meta-analyses, seizure recurrence rates in untreated patients range from $39 \%$ to $51 \%[1,2,6,7]$ at a 2-year followup. In some cases, these data may not justify immediate treatment, but other studies have looked at variables that increase the risk of recurrence $[7,8,10]$. Abnormalities on routine EEG testing and/or symptomatic causes of epilepsy, usually identified by brain imaging, increase the risk of seizure recurrence [8]; these are important considerations when deciding to initiate antiepileptic therapy.

Regardless of the odds of seizure recurrence, the decision to initiate treatment is ultimately an individualized matter. Variables such as work status, dependence on driving, comorbid medical conditions, and concerns about medication side effects and idiosyncratic reactions all play an important role in tailoring a decision for a particular patient.

\section{Deciding to Discontinue an AED}

The decision to discontinue treatment with an AED requires consideration of the same variables taken into account in deciding to start treatment. The patient plays a key role in this decision-making. Some patients are willing to stop AEDs even when the risk of relapse is substantial, whereas others fear the effects of seizure recurrence on quality of life and opt to continue AEDs.

With the publication of the landmark Kwan and Brodie study [11], physicians recognized that approximately two thirds of epilepsy patients could achieve seizure freedom when treated with an appropriately chosen AED. Though these data are certainly favorable for most patients with new-onset epilepsy, they lead to a difficult question for some: After achieving seizure remission, is it appropriate to withdraw AED therapy?

As one may expect, there is no definitive answer. Important historical and recent studies provide some data to guide an approach to this question, but ultimately, population-based odds of recurrence serve only as a starting point for each individual's situation. Most patients do not fit perfectly into a study population, so multiple unique variables relevant to the individual must be carefully considered.

The question of withdrawing AEDs after a seizure-free interval has been debated for many years. A 1994 metaanalysis by Berg and Shinnar [12,Class IV] demonstrated relapse risks of $25 \%$ at 1 year and $29 \%$ at 2 years after AED discontinuation. Most studies have examined patients who were seizure-free for periods of 2 or 4 years. Recurrence of seizures in these populations seemed to occur relatively soon after AED discontinuation, with about half of recurrences occurring within 6 months. The entire recurrence risk due to discontinuation was seen within the first 2 years.

In 1996, the American Academy of Neurology (AAN) recognized the importance of this frequent clinical dilemma and issued a practice parameter [13]. Using a system of evidence classification, the parameter identified 17 studies for inclusion, although only one study met the class I requirements of a randomized controlled trial [14]. The parameter further divided the included studies into categories examining children versus adults. The seizure recurrence rates after AED discontinuation were then reported as pooled, weighted averages: $31.2 \%$ for children and $39.4 \%$ for adults [13]. Using data from all the included studies, the authors also identified several variables that suggested a greater chance of AED withdrawal without relapse: a seizure-free period on AEDs of 2 to 5 years, a single reported seizure type, a normal neurologic exam and IQ, and a normalized EEG with treatment. The guideline concluded that both pediatric and adult epilepsy patients with these characteristics should be offered the option of discontinuing antiepileptic therapy [13].

Adult Studies The AAN practice parameter also highlighted the need for more randomized, double-blind, controlled prospective trials to better assess this situation. At that time, only one unblinded randomized controlled trial had been conducted in the adult epilepsy population. This study, conducted by the Medical Research Council [MRC] Antiepileptic Drug Withdrawal Study Group, and was published in 1991 [14,Class II]. The primary criterion for inclusion was a seizure-free period of 2 years on AEDs, and 1013 patients were randomized to either continued drug treatment or a slow withdrawal over 6 months. At 2 years of follow-up, the continued-treatment group had a relapse rate of $22 \%$, compared with a relapse rate of $41 \%$ in the withdrawal 
group. The hazard ratio (seizure recurrence rate with withdrawal divided by the seizure recurrence rate with continuation) peaked at 9 months and continued to demonstrate a higher risk in the withdrawal group at 2 years. After 2 years of follow-up, however, the hazard ratio inverted, and the withdrawal group no longer exhibited a higher rate of recurrence.

In 2002, Specchio and colleagues [15, Class II] published another prospective study of 330 epilepsy patients who had been seizure-free on monotherapy for at least 2 years. These patients were not randomized but were divided into two cohorts based on those who chose a drug withdrawal option. Those patients who entered the discontinuation arm had their medication tapered over a 3-month period. The continuedtreatment cohort had a relapse rate of $28 \%$, compared with a rate of $50 \%$ for the withdrawal cohort. Mean follow-up times were 48.0 months for the continuation group and 46.6 months for the withdrawal group. Overall, this study reported a seizure relapse risk in the discontinuation group that was 2.9 times that of the treated group, even after accounting for prognostic factors.

Aktekin and colleagues [16, Class III] published another prospective but unrandomized study in 2006, exploring the seizure relapse rate in adult patients who had been seizure-free for at least 4 years. The discontinuation program eventually enrolled 49 patients, with medication being tapered by one sixth every 2 months. Follow-up was 36 months, during which the risk of seizure relapse was $52 \%$. The highest rate of relapse occurred early in the drug withdrawal period [16].

In 2008 , Lossius and colleagues [17•,Class I] published the first randomized, double-blind, controlled trial of AED withdrawal in adult epilepsy patients who had been seizure-free for at least 2 years. In this study, 79 patients were randomized to drug withdraw$\mathrm{al}$, and 81 patients entered the drug continuation arm. Both groups were observed for at least 12 months or until seizure relapse. The authors reported a seizure relapse rate of $15 \%$ in the AED withdrawal group, compared with $7 \%$ for the drug continuation group. Further follow-up to a mean of 41 months revealed a relapse rate of $27 \%$ in those patients who discontinued therapy; unfortunately, this longer-term relapse rate cannot be compared with a rate in the continuation group because many of those patients chose to withdraw from medications at the end of the blinded period. Additionally, it is worth noting that potential bias was introduced in this study because it did not in- clude patients with a known higher risk of relapse, such as those with idiopathic generalized epilepsy, epileptiform discharges on EEG, or polytherapy [18].

Factors to Consider in Adults These studies all confirm an increased risk of seizure recurrence following AED withdrawal, even after a period of seizure freedom. For some patients, this may be a risk worth taking. Some special factors may further increase the risk of recurrence, however, and this information should be presented to patients who are considering drug withdrawal. Several reviews of AED withdrawal have identified a number of factors associated with increased risk: an abnormal neurologic examination, IQ less than 70, longer duration of epilepsy, higher number of seizures, multiple seizure types, focal epileptiform abnormalities on EEG, and worsening EEG patterns after AED discontinuation $[9,19]$. Multiple studies have shown that longer duration of disease and earlier age of onset prior to the period of seizure freedom increase recurrence risk $[13,14,15,16]$. The MRC study [14] found that a higher risk of recurrence is correlated with both the prior use of multiple AEDs and generalized tonic-clonic seizure types. Certain epilepsy syndromes, such as juvenile myoclonic epilepsy, are also known to be associated with a higher relapse risk [19]. The randomized controlled trial by Lossius et al. [17•,Class I] linked a normal neurologic examination and a history of carbamazepine use with a lower risk of seizure recurrence, but it should be noted again that this study excluded many patients likely to be at higher risk based on some of the factors listed above.

Some studies have also explored the impact of testing results on the likelihood of a seizure recurrence. Several studies have shown that EEG abnormalities at the time of drug discontinuation increase the risk of relapse in adults $[15,20]$, and this association also has been well demonstrated in the pediatric population [19]. However, the trial by Lossius et al. [17•, Class I] did not find any prognostic correlation with abnormal or epileptiform EEG studies, although this study did not include certain epilepsy syndromes with specific EEG patterns. In addition to EEG abnormalities, focal findings on MRI, such as hippocampal pathology, have been associated with an increased risk of recurrence during drug withdrawal [21].

Pediatric Studies In the pediatric epilepsy population, there are special considerations involved in the deci- 
sion to discontinue medications, and these patients are often appropriately studied separately from adults. The natural course of some pediatric epilepsy syndromes is remission, regardless of treatment [22]. The original practice parameter published by the AAN demonstrated a slightly lower seizure relapse rate in the pediatric population (31\%) than in adults $(39 \%)$ [13].

A 1998 study by Peters et al. [23, Class I] represents a true prospective, randomized controlled trial for AED withdrawal in the pediatric epilepsy population. In this study, children who had achieved seizure freedom on medications were randomized to drug withdrawal after 6 or 12 seizure-free months. The 6-month cohort experienced a relapse rate of 55\%, compared with $49 \%$ for the longer-treatment cohort. The magnitude of these relapse rates is consistent with the findings of the MRC randomized trial [14], as well as with more recent prospective withdrawal trials focusing on the pediatric population $[24,25,26,27,28,29,30]$. As with adults, the data are relatively conclusive in showing that AED withdrawal is associated with a substantially increased risk of seizure recurrence, especially earlier in the time course after the discontinuation.

Factors to Consider in Children As with the adult epilepsy population, a number of variables must be considered for any individual child who has entered a seizure-free period. The relationship between the number of years of seizure freedom and the relapse rate remains a controversial area. The original MRC study [14] suggested that the longer the period of seizure freedom before AED withdrawal, the greater the chances of avoiding a seizure recurrence. This study pertained largely to adults, however, and other studies in the pediatric population have not demonstrated such a clear correlation $[22,25$, 27]. In a 2008 review, Camfield and Camfield [22] recommended a seizure-free period of 1 to 2 years as an acceptable length of time to justify an AED withdrawal trial in the pediatric epilepsy population.

Perhaps more than in adult population, a child's epilepsy syndrome may also play an important role in the decision to withdraw AEDs. Several pediatric epilepsy syndromes are known to naturally reach a period of remission in all cases, whereas others have a more uncertain prognosis [31]. About $10 \%$ of childhood epilepsy syndromes, including syndromes such as juvenile myoclonic epilepsy and reading epilepsy, will never remit, and thus an AED withdrawal trial would not be advisable, even after a period of seizure freedom [22]. Syndromes with an intermediate prognosis include the localization-related epilepsies, which have been shown to have a higher risk of recurrence in multiple studies $[23,24,26,28,30]$. However, a diagnosis of localization-related epilepsy should not uniformly prevent an AED withdrawal trial in seizurefree pediatric patients.

Other factors that have been associated with a higher relapse risk include adolescent age of onset $[12,25]$, a symptomatic etiology $[12,28,30]$, an abnormal neurologic examination $[19,25]$, and an epileptiform EEG pattern before or after withdrawal $[12,23,29,32]$. An early response to the initial AED therapy has been associated with a lower risk of seizure recurrence $[12,19]$.

\section{Risks and Benefits of AED Withdrawal}

The decision to attempt an AED withdrawal does not depend exclusively on the risk of recurrence. For patients who have achieved seizure freedom with therapy, the primary consideration is a careful weighing of the risks and benefits of the medical therapy. The idiosyncratic reactions to AEDs and their short-term and long-term adverse effects are well known. Limiting these effects is highly desirable. To varying degrees, all AEDs can cause symptoms affecting quality of life, such as drowsiness, fatigue, and inattention [33]. Additionally, older AEDs with effects of hepatic enzyme induction or inhibition can prove particularly undesirable for patients taking multiple medications for other conditions.

Several studies have documented improved outcomes on common neuropsychiatric batteries and mood assessment scales following AED discontinuation $[34,35,36]$, further validating concerns about the impact of AED therapy on quality of life. Medications may lead patients to perform less efficiently at work and at home. Finally, there is also a certain stigma attached to needing daily medications. If a medication may not be necessary, it is reasonable-and perhaps even requisite-to offer the patient a trial of medication withdrawal.

Of course, there are also substantial risks associated with AED withdrawal. The obvious and substantial risk that is highlighted in this review is the likelihood of a seizure recurrence. However, it is important to broaden the discussion of this possibility to consider the implications of a relapse. Once a patient has experienced seizure recurrence, is there a risk that the prior 
responsiveness of the epilepsy has changed? Put another way, does the patient risk developing a medically intractable epilepsy after AED withdrawal? Evidence suggests that this is usually not the case. In 2005, Camfield and Camfield [27] reported a prospective childhood epilepsy study that followed patients who had become seizure-free and appropriately attempted drug withdrawal. In their study population, $29 \%$ had a seizure recurrence that required the reintroduction of medications, and $97 \%$ of these patients regained control. Of the entire cohort being followed, less than $1 \%$ developed medically resistant epilepsy after a trial of AED withdrawal.

Studies in adults have been less clear, and a significant number of patients with medically intractable epilepsy retrospectively report a prolonged period of seizure freedom in their past. This situation raises the concern of being "fooled" into attempting AED withdrawal. A 2005 review of uncontrolled epilepsy after a withdrawal trial cites a mean of $19 \%$ of patients developing medically resistant epilepsy [37]. The risk factors associated with a higher likelihood of this scenario were localization-related epilepsy, a symptomatic etiology, and an abnormal neurologic examination. The authors of this review cautioned against AED discontinuation in the adult population, especially when these known risk factors are present.

The potential for death from a seizure or a seizurerelated event is another concern associated with re- lapse. Status epilepticus and sudden unexplained death in epilepsy (SUDEP) are the most serious complications directly related to a seizure. In multiple studies of both pediatric and adult populations, the occurrence of seizure-related fatalities is very low [22] and should not be emphasized in the decision to attempt an AED withdrawal.

Much more likely than the development of intractable epilepsy or death from seizure relapse are negative psychological effects and effects on quality of life. These are certainly important considerations before AED withdrawal. After adjusting to seizure control, a patient may suffer a significant psychological setback because of a recurrence. Driving and occupational duties are often affected by a relapse even if seizure control is rapidly regained; the impact on quality of life may thus be dramatic. For many patients, the mere possibility of such an event is enough to prevent them from attempting an AED withdrawal.

Additionally, the discontinuation of some AEDs may actually worsen psychological or neurologic functioning, particularly in patients who suffer from psychiatric or other comorbidities. Medications such as valproic acid and lamotrigine are also indicated for mood stabilization in patients with bipolar disorder, and topiramate is commonly used in managing headache disorders. Thus, it is important to consider the full ramifications of a withdrawal trial for epilepsy patients with certain comorbidities.

\section{Treatment}

- Once a patient has entered a seizure-free interval and the possibility of withdrawing medication is considered, it is vital to discuss the appropriate time to initiate the withdrawal and the mechanism for doing so. Unfortunately, there is no clear consensus on these topics for either adults or children. Withdrawal studies in children have quoted optimum seizure-free periods of 1 to 2 years [12], but some of the studies on which these data are based have methodologic shortcomings. Nonetheless, the Camfield and Camfield discussion piece in 2008 [22] asserts that a seizure remission period of 2 years is reasonable before attempting a drug withdrawal in children.

- The authors of a Cochrane review published in 2010 (re-analyzed after a similar 2001 review) took a systematic approach to this issue, searching for randomized controlled trials that addressed the length of seizure remission [38•]. The objective of the review was to compare relapse risk after early ( $<2$ years) and late $(>2$ years) AED 
withdrawal. For the pediatric epilepsy population, the authors analyzed seven controlled trials, determining that the evidence supports a seizure-free period of 2 years before attempting a discontinuation trial in children [38•]. In this review, the authors also found that localization-related epilepsy or an abnormal EEG predicted a higher rate of relapse in those patients who attempted an early drug withdrawal.

- In this same Cochrane review, the authors found no eligible trials that examined seizure-free adults, further validating the need for further research in this area. The original AAN practice parameter from 1996 suggested a wide range of 2 to 5 years of seizure freedom prior to a withdrawal trial [13], and the MRC study [14] required a period of at least 2 years' remission for entry into the study. The 2008 review by Camfield and Camfield [22] suggests at least a 4-year window of seizure remission for adults. These conclusions are based on less rigorous (often retrospective) analyses in adults; it is most accurate to state that evidence is lacking in this area. There are no data supporting the withdrawal of AEDs prior to 2 years of remission in adults.

- Relatively few studies have focused on the mechanism for withdrawing AEDs. The choice is between a relatively rapid taper period or a slow taper period. Intuitively, it is inappropriate to abruptly discontinue any AED because of an increased risk of withdrawal seizures or even status epilepticus. This principle is particularly true for benzodiazepines and barbiturates. Beyond this recommendation, there is little evidence to support a particular style of medication withdrawal.

- In the 2010 Cochrane review [38•,Class I], the range of tapering period for pediatric studies was from 4 weeks to 1 year. A 1994 study compared withdrawal tapers of 6 weeks and 9 months in children with epilepsy, finding no difference in seizure recurrence [24]. More recently, a 2005 paper reported the results of a prospective, randomized trial of medication taper periods of 1 month versus 6 months in children; there was no difference between the two methods in the overall seizure recurrence rate [39].

- For adult epilepsy patients, there is little evidence to guide practice in this area. In 2006, a Cochrane review attempted to identify controlled trials in adults that addressed the question of tapering speed [40]. The authors defined a "slow" taper as occurring over more than 3 months, whereas a "rapid" taper was defined as occurring within 3 months or less. Unfortunately, the authors could identify no adult studies that met their methodologic criteria, further highlighting the lack of evidence in this area. In the absence of definitive data, quality-of-life and safety issues may favor relatively rapid withdrawal (1-3 months). Because most seizures occur in the 
first 6 months after AED discontinuation, a protracted withdrawal schedule extends the maximal "at risk" period for relapse, during which the patient may need to restrict activities such as driving or working around equipment with moving parts.

- The population of epilepsy patients who have undergone surgical resection deserves special mention regarding the decision to discontinue AEDs. For patients who achieve a prolonged period of seizure remission after epilepsy surgery, the standard of care can vary widely, but many physicians apply the same 2-year requirement for this group [41]. A 2004 study published by McIntosh et al. [42,Class IV] supports this practice to some extent; their study followed 325 temporal lobectomy patients for as long as 10 years. They demonstrated that the rate of seizure freedom declined over this length of follow-up, but the discontinuation of AEDs after 2 years of remission was not correlated with the increased recurrence. In 2006, a study by Berg and colleagues [43,Class IV] showed similar results in a group of postsurgical patients who had been seizure-free for at least 1 year. Again, although the risk of seizure recurrence increased over the length of follow-up, the evidence showed that this risk was independent of whether AEDs were withdrawn. In 2009, Kerling et al. [44,Class II] published a prospective, randomized study of postsurgical patients who were seizure-free for 1 year; this study also detected no differences in the rate of recurrence. These studies fully support the practice of undergoing a withdrawal trial after 1 to 2 years of seizure remission following surgery.

- As with patients who have not undergone epilepsy surgery, there are certain factors that elevate the recurrence risk in patients after surgery. Several studies have demonstrated an increased risk in patients with a longer duration of epilepsy, a history of secondarily generalized seizures, and an absence of brain pathologic findings [42,43,44,45]. Patients with identifiable hippocampal sclerosis or other known lesions have a greater chance of successfully maintaining seizure freedom after surgery [42]. Patients who underwent neocortical resections have been shown to do worse than those undergoing standard anterior temporal lobectomy $[46,47]$, but some of those patients have successfully withdrawn from medications [46].

- In a 2008 commentary, Cole and Wiebe [48•,Class IV] discussed the true nature of the evidence supporting the practice of AED withdrawal in postsurgical patients. After conducting a literature search, they applied the same criteria for level of evidence that were used by the AAN. Of 24 identified studies relevant to the issue, none reached a level of evidence greater than class IV, which would not justify clinical recommendation by the AAN standards. This situation highlights the need for further research in this area, but it should not necessarily prevent patients from attempting AED withdrawal trials. 
No potential conflicts of interest relevant to this article were reported.

\section{Open Access}

This article is distributed under the terms of the Creative Commons Attribution Noncommercial License which permits any noncommercial use, distribution, and reproduction in any medium, provided the original author(s) and source are credited.

\section{References and Recommended Reading}

Papers of particular interest, published recently, have been highlighted as:

- Of importance

$\bullet \quad$ Of major importance

1. Berg AT, Shinnar S: The risk of seizure recurrence following a first unprovoked seizure: a quantitative review. Neurology 1991, 41:965-972.

2. First Seizure Trial Group: Randomized clinical trial of the efficacy of antiepileptic drugs in reducing the risk of relapse after a first unprovoked tonic-clonic seizure. Neurology 1993, 43:478-483.

3. Hauser WA, Rich SS, Lee JR, et al.: Risk of recurrent seizures after two unprovoked seizures. $N$ Engl J Med 1998, 338:429-434.

4. Ramos-Linzana J, Cassinello GE, Carrasco LL, et al.: Seizure recurrence after a first unprovoked seizure in childhood: a prospective study. Epilepsia 2000, 41:1005-1013.

5. O'Dell C, Shinnar S: Initiation and discontinuation of antiepileptic drugs. Neurol Clin 2001, 19:289-311.

6. Marson A, Jacoby A, Johnson A, et al.: Immediate versus deferred antiepileptic drug treatment for early epilepsy and single seizures: a randomized controlled trial. Lancet 2005, 365:2007-2013.

7. Kim LG, Johnson TL, Marson AG, et al.: Prediction of risk of seizure recurrence after a single seizure and early epilepsy: further results from the MESS trial. Lancet Neurol 2006, 5:317-322.

8. Berg AT: Risk of recurrence after a first unprovoked seizure. Epilepsia 2008, 49:13-18.

9. Shih JJ, Ochoa JG: Systematic review of antiepileptic drug initiation and withdrawal. Neurologist 2009, 15:122-131.

10. Schreiner A, Phlmann-Eden B: Value of the early electroencephalogram after a first unprovoked seizure. Clin Electroencephalogr 2003, 34:140-144.

11. Kwan P, Brodie MJ: Early identification of refractory epilepsy. N Engl J Med 2000, 342:314-319.
12. Berg AT, Shinnar S: Relapse following discontinuation of antiepileptic drugs: a meta-analysis. Neurology 1994, 44:601-608.

13. American Academy of Neurology: Practice parameter: a guideline for discontinuing antiepileptic drugs in seizure-free patients-summary statement. Neurology 1996, 47:600-602.

14. Medical Research Council Antiepileptic Drug Withdrawal Study Group: Randomised study of antiepileptic drug withdrawal in patients in remission. Lancet 1991, 337:1175-1180.

15. Specchio LM, Tramacere L, La Neve A, et al.: Discontinuing antiepileptic drugs in patients who are seizure-free on monotherapy. J Neurol Neurosurg Psychiatry 2002, 72:22-25.

16. Aktekin B, Dogan EA, Oguz Y, et al.: Withdrawal of antiepileptic drugs in adult patients free of seizures for 4 years: a prospective study. Epilepsy Behav 2006, 8:616-619.

17. Lossius MI, Hessen E, Mowinckel P, et al.: Consequences of antiepileptic drug withdrawal: a randomized, double-blind study. Epilepsia 2008, 3:455-463.

This recent study is the first randomized, double-blind, controlled trial of AED withdrawal in adult epilepsy patients who had been seizure-free for at least 2 years. Although the study has limitations in terms of patient selection criteria and length of follow-up, it is an important addition to the prior studies examining seizure recurrence after drug discontinuation.

18. Schmidt D: Antiepileptic drug withdrawal in seizure-free patients. Lancet 2008, 372:610-611.

19. Specchio LM, Beghi E: Should antiepileptic drugs be withdrawn in seizure-free patients? CNS Drugs 2004, 4:201-212.

20. Callaghan N, Garrett A, Goggin T: Withdrawal of anticonvulsant drugs in patients free of seizures for 
two years. A prospective study. N Engl J Med 1988, 318:942-946.

21. Cardoso TAM: Hippocampal abnormalities and seizure recurrence after antiepileptic drug withdrawal. Neurology 2006, 67:134-136.

22. Camfield P, Camfield C: When is it safe to discontinue AED treatment? Epilepsia 2008, 49(Suppl 9):25-28.

23. Peters ACB, Brouwer OF, Geerts AT, et al.: Randomized prospective study of early discontinuation of antiepileptic drugs in children with epilepsy. Neurology 1998, 50:724-730.

24. Tennison M, Greenwood R, Lewis D, et al.: Discontinuing antiepileptic drugs in children with epilepsy: a comparison of a six-week and a nine-month taper period. N Engl J Med 1994, 330:1407-1410.

25. Verrotti A, Morresi S, Basciani F, et al.: Discontinuation of anticonvulsant therapy in children with partial epilepsy. Neurology 2000, 55:1393-1395.

26. Geerts AT, Niermeijer JM, Peters AC, et al.: Four-year outcome after early withdrawal of antiepileptic drugs in childhood epilepsy. Neurology 2005, 64:2136-2138.

27. Camfield P, Camfield C: The frequency of intractable seizures after stopping AEDs in seizure-free children with epilepsy. Neurology 2005, 64:973-975.

28. Sillanpaa M, Schmidt D: Prognosis of seizure recurrence after stopping antiepileptic drugs in seizure-free patients: a long-term population-based study of childhood-onset epilepsy. Epilepsy Behav 2006, 8:713-719.

29. Olmez A, Arslan U, Turanli G, et al.: Risk of recurrence after drug withdrawal in childhood epilepsy. Seizure 2009, 18:251-256.

30. Ramos-Lizana J, Aguirre-Rodriguez J, Aguilera-Lopez P, Cassinello-Garcia E: Recurrence risk after withdrawal of antiepileptic drugs in children with epilepsy: a prospective study. Eur J Paediatr Neurol 2010, 14:116-124.

31. Camfield PR, Camfield CS: Childhood epilepsy: what is the evidence for what we think and what we do? J Child Neurol 2003, 18:272-287.

32. Andersson T, Braathen G, Persson A, et al.: A comparison between one and three years of treatment in uncomplicated childhood epilepsy: a prospective study. The EEG as predictor of outcome after withdrawal of treatment. Epilepsia 1997, 38:225-232.

33. St. Louis EK: Minimizing AED adverse effects: improving quality of life in the interictal state in epilepsy care. Curr Neuropharm 2009, 7:106-114.

34. Jacoby A: Psychosocial outcomes of antiepileptic drug discontinuation. Epilepsia 1992, 33:1123-1131.

35. Hessen E, Lossius MI, Reinvang I, Gjerstad L: Influence of major antiepileptic drugs on neuropsychological function: results from a randomized, double-blind, placebo-controlled withdrawal study of seizure-free epilepsy patients on monotherapy. J Int Neuropsychol Soc 2007, 13:393-400.
36. Lossius HE, MI RI, Gjerstad L: Slight improvement in mood and irritability after antiepileptic drug withdrawal: a controlled study in patients on monotherapy. Epilepsy Behav 2007, 10:449-455.

37. Schmidt D, Loscher W: Uncontrolled epilepsy following discontinuation of antiepileptic drugs in seizure-free patients: a review of current clinical experience. Acta Neurol Scand 2005, 111:291-300.

38. Sirven J, Sperling MR, Wingerchuk DM: Early versus late antiepileptic drug withdrawal for people with epilepsy in remission. The Cochrane Library 2010, Issue 4.

This study used the Cochrane database to assess the effect of length of seizure remission on outcome following AED withdrawal. The paper concluded for pediatric patients that a period of 2 years seizure-free was appropriate (Class I). However, no Class I studies were found for the adult population, making a determination difficult.

39. Serra JG, Montenegro MA, Guerreiro MM: Antiepileptic drug withdrawal in childhood: Does the duration of tapering off matter for seizure recurrence? J Child Neurol 2005, 20:624-626.

40. Ranganathan LN, Ramaratnam S: Rapid versus slow withdrawal of antiepileptic drugs. Cochrane Database Syst Rev 2006, 2:CD005003.

41. Berg AT, Langfitt JT, Spencer SS, Vickrey BG: Stopping antiepileptic drugs after epilepsy surgery: a survey of U.S. epilepsy center neurologists. Epilepsy Behav 2007, 10:219-222.

42. McIntosh AM, Kalnins RM, Mitchell LA, et al.: Temporal lobectomy: long-term seizure outcome, late recurrence and risks for seizure recurrence. Brain 2004, 127:2018-2030.

43. Berg AT, Vickrey BG, Langfitt JT, et al.: Reduction of AEDs in postsurgical patients who attain remission. Epilepsia 2006, 47:64-71.

44. Kerling F, Pauli E, Lorber B, et al.: Drug withdrawal after successful epilepsy surgery: How safe is it? Epilepsy Behav 2009, 15:476-480.

45. Yoon HH, Kwon HL, Mattson RH, et al.: Long-term seizure outcome in patients initially seizure-free after resective epilepsy surgery. Neurology 2003, 61:445-450.

46. Park KI, Lee SK, Chu K, et al.: Withdrawal of antiepileptic drugs after neocortical epilepsy surgery. Ann Neurol 2010, 67:230-238.

47. Engel J, Van Ness PC, Rasmussen TB, et al.: Outcome with respect to epileptic seizures. In Surgical Treatment of the Epilepsies. By Engel J. New York: Raven Press; 2003:609-621.

48. Cole AJ, Wiebe S: Debate: Should antiepileptic drugs be stopped after successful epilepsy surgery? Epilepsia 2008, 49(Suppl 9):29-34.

This review article debates the risks and benefits of attempting AED discontinuation after successful epilepsy surgery. The authors present the current state of evidence for the specific situation of seizure remission following surgery, highlighting a lack of rigorous scientific methodology. 